\title{
A NOTE ON ANTI-PLANE SHEAR FOR COMPRESSIBLE MATERIALS IN FINITE ELASTOSTATICS
}

\author{
JAMES K. KNOWLES
}

(Received 15 November 1976)

\begin{abstract}
This note gives a necessary and sufficient condition that a compressible, isotropic elastic material should admit non-trivial states of finite anti-plane shear.
\end{abstract}

One of the simplest classes of deformations of solids is that of anti-plane shear, in which each particle of a cylindrical body undergoes a displacement parallel to the generators of the cylinder and independent of the axial position of the particle. Problems involving deformations of this kind are often helpful in the study of qualitative effects whose analogues in more elaborate deformations such as plane strain may be much less accessible because of technical complexities. Because of their utility in this respect, anti-plane shear fields have proved to be especially instructive in crack problems of the type arising in fracture mechanics. (Examples may be found in the references given in [6]).

In the linearized theory of elasticity, non-trivial equilibrium fields of anti-plane shear are possible in the absence of body forces for any homogeneous, isotropic material. In contrast, the exact theory of anti-plane shear in finite elasticity is marred slightly by the fact that not all such materials have this property. This inference is implicit in the work of Adkins [1] for incompressible elastic materials. (See Section 2.20 of [4] for a summary of the analysis in [1].) Much of his study, which appears to have been the first detailed investigation of anti-plane shear in finite elastostatics, is limited to the so-called Mooney material which does admit non-trivial states of anti-plane shear and which is of especial interest in the theory of rubber elasticity.

In [6] a necessary and sufficient condition that an incompressible, isotropic elastic material should sustain non-trivial states of finite anti-plane shear was given in terms of the strain energy density characteristic of the material. In this note an analogous condition is established for compressible materials. JOURNAL may be reproduced by any process without written permission from the Treasurer of the Australian Mathematical Society. 
Consider a homogeneous, isotropic elastic body which in its unstressed, undeformed state occupies a cylindrical region $\mathscr{R}$ whose generators are parallel to the $x_{3}$-axis of a rectangular cartesian coordinate system. A deformation in which a particle with coordinates $\dagger x_{i}$ in the undeformed state has coordinates $y_{i}=y_{i}\left(x_{1} x_{2}, x_{3}\right)$ in the deformed configuration is called an anti-plane shear if

$$
y_{1}=x_{1}, \quad y_{2}=x_{2}, \quad y_{3}=x_{3}+u\left(x_{1}, x_{2}\right),
$$

where the out-of-plane displacement $u$ is regarded as a function on a cross-section $\mathscr{D}$ of $\mathscr{R}$. The deformation gradient matrix $\mathbf{F}$ associated with (1) is given by

$$
\mathbf{F}=\left(y_{i, j}\right)=\left(\begin{array}{ccc}
1 & 0 & 0 \\
0 & 1 & 0 \\
u, 1 & u, 2 & 1
\end{array}\right),
$$

while the left Cauchy-Green deformation matrix is

$$
\mathbf{G}=\mathbf{F F}^{\mathbf{T}}=\left(\begin{array}{ccc}
1 & 0 & u_{, 1} \\
0 & 1 & u, 2 \\
u_{, 1} & u_{32} & 1+|\nabla u|^{2}
\end{array}\right),
$$

where $\mathbf{F}^{\mathrm{T}}$ is the transpose of $\mathbf{F}$, and

$$
|\nabla u|^{2}=u_{\alpha_{\alpha}} u_{\gamma_{\alpha}} .
$$

The fundamental invariants of $\mathbf{G}$ are defined by

$$
I_{1}=\operatorname{Tr} \mathbf{G}, \quad I_{2}=\frac{1}{2}\left[(\operatorname{Tr} \mathbf{G})^{2}-\operatorname{Tr}\left(\mathbf{G}^{2}\right)\right], \quad I_{3}=\operatorname{det} \mathbf{G} ;
$$

for the anti-plane shear (1) they take the form

$$
I_{1}=I_{2}=3+|\nabla u|^{2}, \quad I_{3}=1 .
$$

The stress-deformation relation for a compressible, homogeneous, isotropic elastic material is given in terms of the strain energy $W=W\left(I_{1}, I_{2}, I_{3}\right)$ per unit undeformed volume which is characteristic of the particular material considered. If $\sigma$ represents the matrix of components of nominal-or Piola-stress (force per unit undeformed area), one has for any deformation (see equations (43A.3), (47.8), and (86.9) of [7]):

$$
\boldsymbol{\sigma}=2 \frac{\partial W}{\partial I_{1}} \mathbf{F}+2 \frac{\partial W}{\partial I_{2}}\left(I_{1} \mathbf{1}-\mathbf{G}\right) \mathbf{F}+2 I_{3} \frac{\partial W}{\partial I_{3}}\left(\mathbf{F}^{\mathrm{T}}\right)^{-1},
$$

where $\left(F^{T}\right)^{-1}$ is the inverse of $F^{T}$.

† Latin subscripts take the values $1,2,3$, while Greek subscripts have the range 1,2 . Repeated subscripts are summed over the appropriate range. A subscript preceded by a comma designates differentiation with respect to the corresponding $x$-coordinate. 
For those special deformations such as (1) which locally preserve volume, one has $I_{3} \equiv 1$; it is convenient in such a case to introduce the notation

$$
\begin{aligned}
W_{0}\left(I_{1}, I_{2}\right) & \equiv W\left(I_{1}, I_{2}, I\right), \\
p\left(I_{1}, I_{2}\right) & \equiv-2 \frac{\partial W}{\partial I_{3}}\left(I_{1}, I_{2}, 1\right),
\end{aligned}
$$

so that when $I_{3}=1,(7)$ may be written in the form

$$
\sigma=2 \frac{\partial W_{0}}{\partial I_{1}} \mathbf{F}+2 \frac{\partial W_{0}}{\partial I_{2}}\left(I_{1} \mathbf{1}-\mathbf{G}\right) \mathbf{F}-p\left(\mathbf{F}^{\mathbf{T}}\right)^{-\mathbf{1}}
$$

This form of the constitutive law-valid only for locally volume-preserving deformations such as (1)-is formally identical with the relation between $\sigma$ and $\mathbf{F}$ for an incompressible isotropic, homogeneous elastic material with strain energy density $W_{0}\left(I_{1}, I_{2}\right)$. In the latter case, however, $p$ is an arbitrary hydrostatic pressure and is not related $a$ priori to the deformation, whereas at present $p$ is linked to $u$ through (9), (6).

When (2), (3) and (6) are substituted into (10), the resulting components of nominal stress are

$$
\begin{gathered}
\sigma_{\alpha \beta}=\left[2 \frac{\partial W_{0}}{\partial I_{1}}+2\left(2+|\nabla u|^{2}\right) \frac{\partial W_{0}}{\partial I_{2}}-p\right] \delta_{\alpha \beta}-2 \frac{\partial W_{0}}{\partial I_{2}} u_{, \alpha} u_{, \beta}, \\
\sigma_{\alpha 3}=\left(-2 \frac{\partial W_{0}}{\partial I_{2}}+p\right) u_{\alpha,}, \\
\sigma_{3 \alpha}=2\left(\frac{\partial W_{0}}{\partial I_{1}}+\frac{\partial W_{0}}{\partial I_{2}}\right) u_{, \alpha}, \\
\sigma_{33}=2 \frac{\partial W_{0}}{\partial I_{1}}+4 \frac{\partial W_{0}}{\partial I_{2}}-p .
\end{gathered}
$$

In these formulas $p$ is given by (9), (6), and $I_{1}, I_{2}$, which occur as the arguments of $W_{0}$, are expressed in terms of $u$ through (6).

The true (or Cauchy) stress matrix $\tau$ is related to $\sigma$ and $F$ through (see equation (43A.3) of [7])

$$
\tau=I_{\mathbf{3}}^{-1} \sigma \mathbf{F}^{\mathbf{T}}
$$

expressions for the $\tau_{i j}$ 's in terms of $u$ may be found from this relation and (2), (6) and (11)-(14).

Since body forces are absent, the differential equations of equilibrium are

$$
\sigma_{i j, j}=0 \text { on } \mathscr{R} \text {. }
$$


When (11)-(14) are substituted into (15) and account is taken of the fact that all quantities are independent of $x_{3}$, there follows

$$
\begin{aligned}
& q,_{\alpha}+\left(2 \frac{\partial W_{0}}{\partial I_{2}} u_{\alpha} u,_{\beta}\right), \beta=0 \text { on } \mathscr{D}, \\
& {\left[2\left(\frac{\partial W_{0}}{\partial I_{1}}+\frac{\partial W_{0}}{\partial I_{2}}\right) u_{, \beta}\right]_{, \beta}=\hat{0} \text { on } \mathscr{\mathscr { D }},}
\end{aligned}
$$

where the abbreviation

$$
q=p-2 \frac{\partial W_{0}}{\partial I_{1}}-2\left(2+|\nabla u|^{2}\right) \frac{\partial W_{0}}{\partial I_{2}}
$$

has been introduced.

In view of the fact that $q$ is expressed in terms of $u$ through (18), (9) and (6), the system (16), (17) actually consists of three differential equations for the single unknown function $u$. Without some restriction on $W$, one would thus expect that only very special solutionst of (17) would also satisfy (16). In order to assure that all solutions of the axial equilibrium equation (17) will be available for use in the analysis of boundary value problems, it is natural to say that a material characterized by the strain energy density $W\left(I_{1}, I_{2}, I_{3}\right)$ admits non-trivial states of anti-plane shear if, for every domain $\mathscr{D}$, every solution $\ddagger$ of (17) also satisfies (16). The theorem which follows furnishes a necessary and sufficient condition on $W\left(I_{1}, I_{2}, I_{3}\right)$ for a material to have this property.

It will be assumed that $W\left(I_{1}, I_{2}, I_{3}\right)$ is three times continuously differentiable and is such that the auxiliary function $W_{0}\left(I_{1}, I_{2}\right)$ defined in (8) satisfies the inequality

$$
\frac{d}{d R}\left[R\left(\frac{\partial W_{0}}{\partial I_{1}}+\frac{\partial W_{0}}{\partial I_{2}}\right)_{I_{1}=I_{2}=3+R^{2}}\right]>0
$$

for all $R \geqslant 0$. For a fixed $R \geqslant 0,(19)$ assures that the quasilinear partial differential equation (17) is elliptic $\$$ at a solution $u$ and at a point $\left(x_{1}, x_{2}\right)$ in $\mathscr{D}$ for which $\left|\nabla u\left(x_{1}, x_{2}\right)\right|=R$; it is thus assumed that (17) is elliptic at every point in $\mathscr{D}$ for every solution $u$.

THEOREM. If the strain energy density $W\left(I_{1}, I_{2}, I_{3}\right)$ is such that (19) holds, then the associated elastic material admits non-trivial states of anti-plane shear if and only if $W$

† One choice of $u$ which satisfies both (16) and (17) for every choice of $W$ is that corresponding to simple shear: $u=k_{\alpha} x_{\alpha}$, where the $k_{\alpha}$ are constants. It can be shown that, apart from an additive constant, this is the only out-of-plane displacement field with this property.

$\ddagger u$ is assumed three times continuously differentiable.

§ See [3], pp. 163-164. 
also satisfies the two conditions

$$
b \frac{\partial W}{\partial I_{1}}+(b-1) \frac{\partial W}{\partial I_{2}}=0 \text { for } I_{1}=I_{2} \geqslant 3, I_{3}=1
$$

$\frac{\partial^{2} W}{\partial I_{1}^{2}}+I_{1} \frac{\partial^{2} W}{\partial I_{1} \partial I_{2}}+\left(I_{1}-1\right) \frac{\partial^{2} W}{\partial I_{2}^{2}}+\frac{\partial^{2} W}{\partial I_{1} \partial I_{3}}+\frac{\partial^{2} W}{\partial I_{2} \partial I_{3}}+\frac{1}{2} \frac{\partial W}{\partial I_{2}}=0$ for $I_{1}=I_{2} \geqslant 3, I_{3}=1$

for some constant $b$.

Proof. To establish the necessity of (20), (21), suppose first that, for any domain $\mathscr{D}$, every solution $u$ of (17) also satisfies (16), with $q$ given in terms of $u$ by (18), (9) and (6). The system (16), (17)-with the relation between $q$ and $u$ deleted-is identical with the system which arises in the incompressible case and which was treated in detail in [6]. $\dagger$ In particular it was shown in [6] $\ddagger$ that if, for every $\mathscr{D}$, there corresponds to each solution $u$ of (17) some function $q$ such that (16) holds, then $W_{0}\left(I_{1}, I_{2}\right)$ necessarily satisfies

$$
b \frac{\partial W_{0}}{\partial I_{1}}+(b-1) \frac{\partial W_{0}}{\partial I_{2}}=0 \text { for } I_{1}=I_{2} \geqslant 3
$$

for some constant $b$. It follows immediately that (22) holds in the present circumstances; reference to (8) shows that (22) is identical with (20), and thus the necessity of (20) is established. Next, one uses (22), together with (17), to write

$$
\begin{aligned}
\left(2 \frac{\partial W_{0}}{\partial I_{2}} u_{\alpha} u_{, \beta}\right)_{, \beta} & =b\left[2\left(\frac{\partial W_{0}}{\partial I_{1}}+\frac{\partial W_{0}}{\partial I_{2}}\right) u_{\alpha} u_{, \beta}\right], \beta \\
& =2 b\left(\frac{\partial W_{0}}{\partial I_{1}}+\frac{\partial W_{0}}{\partial I_{2}}\right) u_{\alpha \beta} u_{, \beta} \\
& =\frac{\partial W_{0}}{\partial I_{2}}\left(u_{, \beta} u_{, \beta}\right)_{\alpha} \\
& =\frac{\partial W_{0}}{\partial I_{2}} 2 R R,
\end{aligned}
$$

where

$$
R=|\nabla u|=(u, \beta, \beta)^{t}
$$

From (18), (9) and (6) it follows that $q$ is a function of $R^{2}$ only: $q=q\left(R^{2}\right)$. Thus

$$
q_{, \alpha}=q^{\prime}\left(R^{2}\right)_{\alpha}=2 R R,_{\alpha} q^{\prime},
$$

$\dagger$ See (3.1)-(3.4) of [6]. For present purposes only that special case of the results in [6] is needed for which axial prestretch is absent. Thus one is to set $\lambda=1$ in (3.1), (3.2) of [6].

$\ddagger$ See (3.22), (3.23) of [6] with $\lambda=1$. Essential use is made of the assumption (19). 
where the prime indicates differentiation with respect to $R^{2}$. Combining (23) and (25) shows that (16) can be written in the form

$$
\left(q^{\prime}+\frac{\partial W_{0}}{\partial I_{2}}\right) 2 R R,_{\alpha}=0 \text { on } \mathscr{D} \text {. }
$$

Now by assumption, (16) and therefore (26) are satisfied whenever $u$ is a solution of (17). Since it is possible to construct a solution of (17) for which, at a given point of $\overline{\mathscr{D}}, K=|\bar{\nabla} u|$ takes an arbitrarily prescribed positive value while $\nabla R \neq 0$, one concludes from (26) that

$$
q^{\prime}\left(R^{2}\right)+\frac{\partial W_{0}}{\partial I_{2}}\left(3+R^{2}, 3+R^{2}\right)=0 \text { for } R \geqslant 0 .
$$

When $q^{\prime}\left(R^{2}\right)$ is expressed in terms of derivatives of $W$ with respect to the invariants by differentiating (18) with respect to $R^{2}$ and making use of (9) and (6), one obtains (21) from (27) immediately. Thus (20) and (21) are indeed necessary.

To show that they are also sufficient, one merely observes that, if $u$ is a solution of (17) for an arbitrary domain $\mathscr{D}$, and if (20) holds, then a review of the above argument shows that (27) implies (16). Since (21) in turn implies (27), (16) is also satisfied, and the proof is complete.

The necessary and sufficient condition of the theorem imposes two restrictions on the strain energy density $W$. In contrast, the corresponding result in [6] for incompressible materials consists of only one requirement which, in fact, is formally identical with (20).

It is possible to show that (20), (21) fail to hold for the strain energy density proposed by Blatz and Ko [2] in connection with experiments on a highly compressible foam rubber, $\uparrow$ and that, apart from degenerate special cases, they fail as well for the class of idealized materials-those of harmonic type-introduced by John. (See Section 6 of [5].) For the so-called Hadamard materials (see p. 313 of [5]) described by a strain energy density of the form $W=A I_{1}+B I_{2}+f\left(I_{3}\right)$, where $A$ and $B$ are constants and $f$ is an essentially arbitrary function, conditions (20), (21) are fulfilled if and only if $B=0$, in which case $b=0$ in (20).

\section{References}

[1] J. E. Adkins, "Some generalizations of the shear problem for isotropic incompressible materials", Proc. Cambridge Philos. Soc. 50 (1954), 334.

[2] P. J. Blatz and W. L. Ko, "Application of finite elastic theory to the deformation of rubbery materials", Trans. Soc. Rheology 6 (1962), 223.

[3] R. Courant and D. Hilbert, Methods of Mathematical Physics, Vol II, Interscience, New York (1962).

[4] A. E. Green and J. E. Adkins, Large Elastic Deformations, Clarendon Press, Oxford (1960).

† See equations (67) and (6), (7), (8) of [2]. 
[5] F. John, "Plane elastic waves of finite amplitude. Hadamard materials and harmonic materials", Communications on Pure and Applied Mathematics 19 (1966), 309.

[6] J. K. Knowles, "On finite anti-plane shear for incompressible elastic materials", J. Aust. Math. Soc. Series B Vol. XIX Part 4, (1976).

[7] C. Truesdell and W. Noll, "The nonlinear field theories of mechanics", Handbuch der Physik, Vol. III/1, Springer, Berlin (1965).

Division of Engineering and Applied Science

California Institute of Technology

Pasadena, California 91125

United States of America 